\title{
Estigma, communitas y modos de corrección para los habitantes de la calle en Bogotá (2000-2010) ${ }^{1}$
}

\section{Stigma, Communitas and Corrective Ways to Homeless People in Bogotá (2000-2010)}

\section{Estigma, communitas e modos de correção para os moradores da rua em Bogotá (2000-2010)}

\section{Carlos José Suárez García ${ }^{2}$}

Universidad de Guadalajara, Guadalajara, México

cjsuarezg@unal.edu.co

Recibido: $22 / 04 / 2016$

Aprobado: $28 / 10 / 2016$

1 Este ensayo se originó como una reflexión de mi disertación de Maestría en Antropología de la Universidad Federal Fluminense (UFF) titulada "El Estado y el abyecto" realizada entre los años 2010 y 2012 (disponible http://www.uff.br/ppga/wp-content/uploads/2013/o6/O-Estado-e-o-Abjeto_Carlos-Jos\%C3\%A9-Suarez-Garcia.pdf).

2 Maestro en Antropología. Maestro en Planeación Urbana y Regional. 


\title{
Resumen
}

Desde una perspectiva simbólica, en este ensayo se reflexiona sobre los modos como la sociedad colombiana contemporánea enfrenta la existencia de los habitantes de la calle. A partir del caso de la calle de El Cartucho y con base en investigaciones realizadas para conocer la oferta institucional para los habitantes de la calle en Bogotá, se ponen a prueba los postulados teóricos de Goffman sobre el estigma y de Turner sobre la communitas. Así, se muestran las representaciones sociales que tienen los funcionarios (gubernamentales, no gubernamentales y religiosos) sobre esta población y su asociación con el peligro y el vicio. Se observan las formas como estas personas consideradas outsiders pasan por un proceso "correctivo", similar a los ritos de paso observados en sociedades orgánicas. Como conclusión, se expone el debate sobre las opciones generales ofrecidas por la sociedad contemporánea a estas personas que habitan la ciudad: "regresarlos a la vida" gracias a las terapias o caer víctima de la "limpieza social".

Palabras clave: habitante de la calle; violencia; control social.

\begin{abstract}
This essay reflects, from a symbolic perspective, about the ways in which the contemporary Colombian society faces up to the existence of homeless people. From the "Calle de El Cartucho" case and based in researches about the institutional offer to homeless people in Bogotá, it tests two theoretical assumptions: the stigma in Erwin Goffman, and the communitas in Victor Turner. In this way, the paper shows the social representations that have about homeless people, governmental, nongovernmental and religious professionals that work with this population, and their association with danger and vice. Then, it observes the ways in which homeless people considered outsiders undergo a "corrective" process, similar to the rites of passage observed in organic societies. Finally, it presents two general options offered by the contemporary society to those homeless people who inhabit the city: the possibility of "return to life" thanks to therapies, or die victims of the "social cleansing".
\end{abstract}

Keywords: Homeless; violence; social cleansing; social control.

\section{Resumo}

Desde uma perspectiva simbólica, neste artigo reflito sobre os modos como a sociedade colombiana contemporânea se defronta com a existência dos moradores de rua. A partir do caso da "calle del Cartucho" e baseado nas pesquisas desenvolvidas para conhecer a oferta institucional para os moradores de rua em Bogotá, examino os postulados teóricos de Goffman sobre o estigma e de Turner sobre a communitas. Assim, apresento as representações sociais que têm os funcionários (governamentais, não-governamentais e religiosos) sobre os moradores de rua, e sua associação com o perigo e o vício. Posteriormente, observo as formas como estas pessoas consideradas "outsiders" passam por um processo "corretivo", similar aos ritos de passagem observados nas sociedades orgânicas. Em conclusão, as opções gerais que a sociedade contemporânea oferece a estas pessoas, se debate entre "retorná-los à vida" mediante terapias, ou deixá-los como vítimas da "limpeza social".

Palavras chave: Moradores de rua; violência; controle social. 


\section{Introducción: el mal y el vicio en la ciudad}

Las representaciones sociales tienen un poder transformador sobre la vida de los seres humanos. A veces somos banales con respecto al uso que hacemos de estas representaciones, y muchas veces no las reconocemos como argumentos justificativos de las acciones reguladoras de la sociedad. Además, la sociedad tiene formas perversas de regularse, de lidiar con la diferencia. Este proceso de regulación es llamado frecuentemente como cruel, sin embargo, esta exclusión debe ser leída como un camino de dos vías: la sociedad puede lanzar fuera a aquellos que no se adaptan a sus principios, o bien puede ser una elección racional de individuos que desean alejarse de las normas de la sociedad. La calle, en este sentido, es uno de esos lugares a donde son lanzados (o a donde saltan) los "desajustados". Son ellos, especialmente los habitantes de la calle, el foco del presente ensayo. No obstante, la reflexión girará en torno a la forma como las personas "normales", especialmente los funcionarios de instituciones que trabajan con niños y adultos habitantes de la calle, representan esa población, para luego observar cuáles son las "estrategias simbólicas" que se siguen para "transformarlos"; entendiendo que "lo simbólico" está necesariamente anclado a una práctica y a una materialidad que se encuentran más allá del plano discursivo. Para reconocer las representaciones sociales de los habitantes de la calle, me centraré principalmente en el caso de la Calle del Cartucho en la capital colombiana, lugar del miedo en la ciudad. La reflexión teórica sobre estos problemas prácticos se basó en investigaciones previas sobre metodologías de atención para habitantes de la calle que realicé en 2006 y 2007, para la Universidad Nacional de Colombia y el Ministerio de Protección Social (UNAL-MinProtección, 2007), y sobre oferta institucional para menores habitantes de la calle para el Instituto Colombiano de Bienestar Familiar (ICBF) y financiada por la Unión Europea (Barrios, Góngora y Suárez, 2006). En estas investigaciones se contactaron los funcionarios de las principales instituciones que atendían esta población en Bogotá (y otras siete ciudades más), fueran gubernamentales, no gubernamentales o religiosas. A pesar de la heterogeneidad de enfoque de atención a estas personas, la representación de los funcionarios tenían elementos en común, que despertaron el interés de esta reflexión, entre estos la mención a la Calle del Cartucho como el lugar donde vivían los habitantes de la calle y cuya destrucción agravó los problemas de seguridad del centro de la ciudad. La relación metonímica entre la calle del Cartucho y los habitantes de la calle, que excluyó la diferencia de las poblaciones en el barrio Santa Inés y que idealizó la destrucción del hábitat de los habitantes de la calle como fórmula de la administración pública para "incidir" sobre la seguridad en el centro de Bogotá, es expuesta con mayor detalle en mi tesis de maestría (Suárez, 2011).

Para observar las representaciones presentaré algunos extractos de entrevistas realizadas a estos funcionarios, algunas de las cuales no fueron publicadas en los informes finales pero que en su momento sirvieron de base para la generación de políticas públicas para esta población. Debido a que la mayoría de las afirmaciones no son "políticamente correctas", menos en el contexto de su "labor social", omito deliberadamente los nombres de los funcionarios. Igualmente, dentro de las investigaciones mencionadas se consideraron las narraciones del periódico 
El Tiempo que, en la actualidad, monopoliza la prensa nacional y moviliza gran parte de la opinión pública del país mediante su red de revistas y sus influencias en los medios masivos de comunicación en general. Como contrapunto, las investigaciones mencionadas (UNAL-MinProtección, 2007; Barrios et al., 2006) se enriquecían con grupos focales y entrevistas para reconocer la percepción de los habitantes de la calle sobre los servicios de atención que prestaban las diferentes entidades. Además de evaluar estos servicios, los propios habitantes de la calle expusieron un abanico de autorrepresentaciones que, si bien no se agotan en este ensayo, coinciden en el miedo que ellos producen en la sociedad, en los "normales". La siguiente reflexión puede enmarcarse entre la desaparición de El Cartucho, un proceso de varios años que inició con la expedición del Decreto 880 de 1998 y que terminó en 2004 con la inauguración del Parque Tercer Milenio (Suárez, 2010), junto con el trabajo de campo entre 2006 y 2007, que comprendió un periodo posterior a este evento, siendo el Cartucho un referente para los funcionarios de instituciones y para los mismos habitantes de la calle.

Luego de realizadas estas investigaciones, quería poner a prueba las tesis de Goffman y de Turner, quienes proveían un soporte para entender las lógicas culturales de las políticas de esta población y de la calle del Cartucho en Bogotá; se trata entonces de probar la eficacia teórica de estas dos corrientes, los llamados "interaccionismo simbólico" y "antropología simbólica", poco utilizados como referentes teóricos en los estudios sobre esta población. Así, una apuesta doblemente arriesgada cuyos resultados pueden no ser "políticamente correctos".

\section{Erwin Goffman y las representaciones sociales}

El análisis de las representaciones sociales, según Erwin Goffman (1975), comienza imaginando el modo como las personas se comportan frente a situaciones nuevas y desconocidas, y cómo obtienen información sobre el nuevo individuo que irrumpe en una situación dada, mediante un "vehículo de indicios", lo que permite desarrollar una elección a partir del abanico de comportamientos posibles. Las informaciones acerca de cómo comportarse frente a un desconocido pueden ser suministradas por la conducta del nuevo individuo y por su apariencia externa. Para Goffman, los primeros indicios pueden estar ligados a las experiencias anteriores de los sujetos, dentro de lo que el autor llama "estereotipos no comprobados". De este modo, gracias a la apariencia de ciertos individuos es posible predecir determinadas formas de comportamiento frente a ellos. Tras la llegada del nuevo individuo a la escena, se sigue lo que Goffman llama una "línea de comportamiento". Una vez iniciada esta "línea de comportamiento", la interacción se desenvuelve entre los participantes con un restringido margen de alteración. He aquí el carácter moral de las proyecciones: las características sociales iniciales (o indiciarias) de un individuo dan a los participantes el derecho de disponer un tratamiento adecuado a su valor social; podría decirse que existe una institucionalización de las expectativas abstractas estereotipadas, en otras palabras, una "representación colectiva".

Para Goffman la calle es el escenario más adecuado para observar este tipo de "rituales cotidianos" gracias a la copresencia coordinada de diferentes actores; en esta arena se harán más visibles las demostraciones de intenciones y 
representaciones de los otros. Tomemos como ejemplo a los habitantes de la calle, el reciclador de basura, el personaje epítome de este "escenario privilegiado", y veremos cómo estas representaciones van más allá de una "interacción simple" o dialógica, para transformarse en una interacción que pretende el cambio total de este individuo despreciado sin "ninguna capacidad de respuesta", o de resistencia, que no sea el mismo hecho de existir. Sin duda, en un primer análisis, estos "excluidos" van a convertirse en "sujetos pasivos de intervención"; pero una observación más atenta permitirá ver cómo suceden otras dinámicas.

La mayoría de los autores e instituciones encargadas de hacer política pública para los habitantes de la calle en la ciudad de Bogotá anotan de forma generalizada que estas poblaciones son consideradas "en riesgo" o "en condición de vulnerabilidad"; el presente ensayo pretende ir más allá, cuestionando esta afirmación para tener en consideración las causas culturales de este riesgo. En las entrevistas realizadas para caracterizar la oferta institucional para "habitantes de la calle" se preguntó por la definición de las personas con quienes trabajaban (UNAL, Minprotección, 2007); estos son algunos apartes no publicados de estas entrevistas, cuyos participantes permanecerán en el anonimato dentro de esta reflexión. La Fundación Renacer tiene como público específico los niños y adolescentes que ejercen la prostitución en la calle, y que viven en situaciones de violencia y exclusión. Para los funcionarios de esta institución "Son personas excluidas socialmente, quienes no existen para el Estado, son desamparados por la sociedad". Los funcionarios de la Asociación Cristiana de Jóvenes (ACJ-YMCA), que actúan junto con adolescentes habitantes de la calle, dicen que ellos son "seres humanos que hacen de la calle su vida, tienen que vivir constantemente entre el consumo [de drogas] y la delincuencia". Las hermanas de la organización religiosa Medalla Milagrosa de San Vicente de Paul afirman que el habitante de la calle "es quien está en estado de exclusión, al margen de la sociedad, sin accesos. Es el desplazado [por la violencia en las zonas rurales], el delincuente, el vicioso de alcohol o de drogas, el homosexual. Todos ellos han llegado aquí [a la Medalla Milagrosa], aun los de cultos satánicos, buscando ayuda. Los habitantes de la calle son personas inmediatistas [que solo piensan en el presente], pero muy transparentes en la amistad". Finalmente, para los miembros del cuerpo médico del Hospital Centro Oriente, que atienden esta población en el centro de la ciudad, el problema de los habitantes de la calle es extensivo y abarca todo el ambiente o hábitat donde ellos permanecen: "La percepción general es que los habitantes de la calle generan inseguridad; se roban el cableado y las tapas de la alcantarillas; crean depresión en los niños que viven cerca de estos sectores marginales porque les da miedo salir a jugar; además insalubridad tanto por basuras, como por sus heces". Finalmente, las palabras de un funcionario del sistema de protección social pueden resumir la representación oficial y generalizada que tienen los equipos que atienden a los habitantes de la calle en Colombia sobre sus propios sujetos de atención:

Yo recuerdo en este momento un autor que decía que los niños de calle tienen una tendencia a animalizarse, o sea, a ser más instintivos, y yo comparto en cierta parte eso. Pienso que la gente que empieza a vivir en la calle desarrolla ese tipo de acciones primarias y entonces los actos violentos se dan entre ellos. Se pueden matar por cualquier pendejada [...]. Existen barrios donde la gente come basura (Barrios et al., 2006, p. 90). 
Tales descripciones traen a la memoria las observaciones hechas por Goffman (1980) sobre el estigma, particularmente aquel caracterizado por el autor como "estigma por las culpas individuales", es decir, aquellas percibidas como voluntad débil, falsas creencias y deshonestidad, normalmente inferidas a partir de los relatos de vicio, desempleo, homosexualidad y alcoholismo. De esta forma, resalta la afirmación del autor de que los portadores de estigmas tienden a tener las mismas creencias sobre sí mismos y su propia identidad que "nosotros, los normales". Por otra parte, así como Goffman lo describe, tales personas estigmatizadas llegan a aproximarse agresivamente a los otros, suscitando respuestas desagradables. No obstante, a veces parece que los habitantes de la calle aprovechan estratégicamente sus estigmas para obtener dinero o comida, mediante la intimidación ostensiva provocada por su "fachada". Una narración del periódico El Tiempo puede ser esclarecedora:

A mediados del año pasado, los ñeros [habitantes de la calle] también intentaron instalarse en el barrio Policarpa ubicado a unas diez cuadras al sur [de la Calle] del Cartucho. Al principio la gente les regalaba comida y ropa, pero se cogieron del codo y comenzaron a formar una ciudadela y a tratar mal a la gente, a cogerles la cola a las muchachas, a quitarles los 500 pesos del helado a los niños y a ofrecerles vicio a los muchachos de los colegios, dice un dirigente comunitario (El Tiempo, 2002).

El trabajo Territorios del miedo en Santafé de Bogotá (Niño, Lugo, Rozo y Vega, 1998) presentaba al habitante de la calle como un "sujeto productor de miedo" entre los ciudadanos de la capital. En este libro aparecen descripciones como la siguiente: "Con su actitud y mirada te dicen te voy a robar"; "tienen la mirada y la apariencia agresiva"; "tienen el pelo enmarañado, engrasado y la ropa en hilachas"; "llevan la ropa tiesa de grasa y la mirada perdida"; "a esos deberían recogerlos porque hacen mucho mal a la humanidad" (Niño et al., 1998, pp. 94-96). El habitante de la calle evoca la pobreza extrema, la suciedad, la delincuencia, el vicio, la degradación, el miedo y el peligro. De la misma forma, en los artículos del periódico El Tiempo se asocia a los habitantes de la calle con la falta de seguridad y el robo en los bienes públicos: "Según la Alcaldía, la ola de delincuencia se extiende, entre otras razones, por el aumento de indigentes, la proliferación de vendedores ambulantes que han vuelto a invadir el sector por actividades como la prostitución" (El Tiempo, 2001). En una entrevista dada al mismo periódico, un dirigente local explicó:

Junto al parque del barrio Policarpa alcanzaron a instalarse unos 15 ñeros en sus carros esferados. Con su presencia aumentó el vandalismo. El año pasado se llevaron como 70 tapas de los contadores del agua, el cable de los teléfonos y unos 20 contadores del gas. Hace tres meses, nosotros cogimos a un tipo con seis tapas en un carro esferado ${ }^{3}$ (El Tiempo, 2002).

Los modos como se denomina entonces al "habitante de la calle" puede ser también significativo para la elaboración de esta representación. Por una parte, tenemos estas descripciones, que mencionan comportamientos relacionados

3 Carritos de balineras usados por los recicladores. 
con el vicio, la delincuencia, la suciedad. Por otra parte, tenemos los nombres "incorrectos" y populares como "gamín", "reciclador", "loco", "bazuquero", o aun "desechable", con que se designa cotidianamente a esta población; estos nombres se contraponen a las largas y "correctas" discusiones si denominarlos, por ejemplo, "menores", "infantes", "jóvenes" o "adolescentes habitantes de la calle". Como veremos más adelante, a la "corrección" esperada de un individuo corresponde una "corrección" en su denominación.

Como contraparte de esta representación generalizada de los habitantes de la calle, tenemos la oportunidad de ver cuáles son las representaciones que los habitantes de la calle tienen de ellos mismos, y comprobaremos la afirmación de Goffman. En el caso de la Calle del Cartucho, lugar que se consideraba el "hábitat" de los habitantes de la calle de Bogotá en la década de 1990, se trata de una representación atravesada por la violencia, el miedo y las drogas. Esto se evidencia, por ejemplo, en los nombres de las monografías de carácter etnográfico y testimonial sobre la Calle del Cartucho: Espacio de la muerte en el Cartucho (Cortés, 1994), Entre el pánico y el éxtasis: territorio en el Cartucho (Martínez, 2000) o Yo salí del infierno: sobreviviente del Cartucho (Rozo y Cadena, 2006). No se trata de abundar en la relación entre violencia y habitantes de la calle en su aspecto intrínseco, es decir, el modo en que la violencia estructura las relaciones dentro del Cartucho y, por extensión, las relaciones "de la calle", explorado en otro artículo (Góngora y Suárez, 2008). En vez de limitarnos al material de investigación de esas monografías, confirmamos mediante un trabajo con los habitantes de la calle cuáles eran sus experiencias de vida, tal vez un ejercicio iterativo que da cuenta de la persistencia de la relación entre la violencia, el miedo y la calle. Para obtener estos testimonios, investigadores de la ciudad de Bogotá realizaron grupos focales en los cuales los habitantes de la calle adultos participaron para hablar de sus vidas. Ellos dijeron que: "Dormir en la calle es feo, uno vive paniquiao [con pánico provocado por el consumo de bazuco], todos borrachos consumidos. En la calle no hay seguridad, todo el mundo lo mira a uno durmiendo y dice 'uy, mírelos". La discriminación es evidentemente percibida por ellos: "Los niños son los que le hacen más visajes a uno, ellos son los que más le dicen a uno los locos". La causa de este comportamiento infantil es también muy clara: "Lo que pasa es que los padres son los que les enseñan a sus hijos a decirle que uno es loco, que uno está enfermo" (UNAL-MinProtección, 2007, pp. 152-153). En resumen, los habitantes de la calle pueden ser leídos simbólicamente según estas representaciones como "seres instintivos", violentos, sucios, caóticos, sin familia y sin ley. Para la mayoría, su sola presencia traerá la delincuencia, la suciedad, la depresión y la drogadicción. $\mathrm{Al}$ representar el epítome de todos los vicios de la sociedad, especialmente la colombiana, son temidos e indeseados.

\section{Victor Turner y la communitas}

Los habitantes de la calle adquieren las características dadas por Victor Turner a la "liminalidad", a la communitas o, como le gusta mencionar a este autor, a la "antiestructura":

Liminality is, of course, an ambiguous state, for social structure, while it inhibits full social satisfaction, gives a measure of finiteness and security; liminality may be for 
many the acme of insecurity, the breakthrough of chaos into cosmos, of disorder into order, then the milieu of creative interhuman or transhuman satisfactions and achievements. Liminality may be the scene of disease, despair, death, suicide, the breakdown without compensatory replacement of normative, well-define social ties and bounds. It may be the anomie, alienation, angst, the three fatal alpha sisters of many modern myths $[. .$.$] in the leisure genres of complex societies; it may be repre-$ sented by the "extreme situations" beloved of existentialist writers: torture, murder, war, the verge of suicide, hospital tragedies, the point of execution, etc. Liminality is both more creative and more destructive than the structural norm (Turner, 1982, pp. $46-47)^{4}$

Aquellos que hacen parte de la communitas o la antiestructura cuestionan la existencia de la propia estructura. Así, ella misma desarrolla mecanismos para mantener estos seres alejados -aquellos que, en el análisis de Van Gennep (1978), son considerados como "muertos" o "peligrosos"-, construyendo a su alrededor muros reales o imaginarios, mediante mecanismos que conservan las fronteras sociales explícitas o implícitas. Este proceso "dialéctico" refuerza los límites de la estructura, aunque en las grandes sociedades exista la tendencia de destruir esta polaridad (Turner, 2008, p. 250). Dentro de la communitas se revela, según Turner, el "verdadero estado de las cosas", donde se hace menos fácil vestir las máscaras, o como diría Goffman (1975), mantener el "trabajo de la fachada". La importancia del trabajo con la margen fue resaltada por Van Gennep (1978) hace ya un siglo, cuando exhortaba que se debía analizar el ritual como un proceso, y no como una secuencia de etapas aisladas. Los rituales no solo pueden ser observados dentro de las iniciaciones, sino también en varias fases de la vida; es por eso que el etnógrafo alemán insiste en la necesidad de percibir cómo se desarrollan los ritos de paso, aunque sus partes no estén igualmente desarrolladas o que su secuencia no sea fijada dentro de un periodo determinado. La persona que entra en la antiestructura queda en suspenso, aun dentro de sus propias reglas; en las sociedades primitivas estudiadas por Van Gennep (1978), el momento de la margen prepara a la persona para su reintegración en la sociedad, con un nuevo papel y responsabilidades sociales que son aprendidas durante la etapa liminar socialmente institucionalizada, un cambio de estatus de la persona y de las cosas, de lo sagrado a lo profano, previsto por la sociedad.

El término communitas usado por Turner es originado a partir del análisis de Arnold Van Gennep (1978) sobre los ritos de paso. Este autor escribió, a inicios del siglo XX, que todo ritual de paso, entre los que se destacan el nacimiento/ bautizo, la pubertad, el matrimonio y la muerte, estaba dividido en tres fases:

4 La liminalidad es, claro está, un estado ambiguo de la estructura social, que mientras inhibe la satisfacción social total, otorga una medida de finitud y seguridad; la liminalidad puede ser para muchos la cima de la inseguridad, la ruptura del caos en el cosmos, el desorden dentro del orden, luego, el medio de las satisfacciones y hazañas interhumanas y transhumanas creativas. La liminalidad puede ser la escena de la enfermedad, la desesperación, la muerte, el suicidio, la ruptura sin reemplazo compensatorio de la normativa, de los bien definidos lazos y límites sociales. Puede ser la anomia, la alienación, la angustia, las tres fatales hermanas alfa de los mitos modernos [...] en el placer de los géneros de las sociedades complejas; puede ser representada como la "situación extrema" tan amada por los escritores existencialistas: tortura, muerte, guerra, el borde del suicidio, tragedias de hospital, puntos de ejecución, etc. La liminalidad es tanto más creativa y más destructiva que la norma estructural. 
separación, margen o liminaridad e integración. Dentro del "momento liminar" el sujeto, que no está acá ni allá -betwixt and between-, es descrito por Van Gennep esencialmente como el neófito, el iniciado o el novicio, aquellos que van a cambiar su estatus social a través del paso, donde la persona encontrará o aprenderá los misterios de su cultura. Pero Turner añade que dentro del estado liminar también se encuentra el outsider (Turner, 2008), aquel que permanece alejado de la estructura, pero que además comparte los atributos de la divinidad: son aquellos los adivinos, los chamanes, los vagabundos y los médiums.

El caso que voy a analizar es de otro tipo de "seres liminares", los marginales, que al contrario de los outsiders sagrados buscan la entrada en el sistema, aunque sin ninguna garantía de resolución final de su estado de ambigüedad. A diferencia de los "liminares rituales", que cambian su estatus original por uno más elevado, los marginales están siempre en decadencia. Para estos seres la sociedad tiene reservada la función simbólica de representar la humanidad, sin calificaciones o características de estatus. En este caso, el más bajo representa la totalidad humana, el caso extremo que retrata más adecuadamente al todo (Turner, 2008, p. 218).

El programa de Turner (2008) es el que estoy siguiendo aquí. Primero, seguir las fases del "drama social": (ruptura $\rightarrow$ crisis $\rightarrow$ acción correctiva $\rightarrow$ reintegración o cisma), que está explícitamente inspirado en el análisis ritual que realizó Van Gennep (1978) hace ya un siglo: (separación $\rightarrow$ margen $\rightarrow$ agregación). Se trata, sobre todo, de prestar mayor atención en la fase correctiva, en la cual se desarrollan y diseminan los mecanismos de ajuste operado por los representantes del orden; si el investigador social tiene interés en el análisis de los cambios, he aquí la recomendación de Turner (2008, p. 36): primero, examinar cuidadosamente lo que sucede en la fase tres; segundo, centrarse en la fase "correctiva" o de "restitución" de los dramas sociales; tercero, preguntarse si la "máquina correctiva" es capaz de hacer frente a esas crisis, de modo que restaure relativamente el status quo anterior, o al menos restaurar la paz entre los grupos contendientes; finalmente, si la "máquina correctiva" no resuelve el drama "reintegrando" a las personas a la sociedad, preguntarse por qué no corrige (Turner, 2008, p. 36). Para Turner (1982), la communitas se convierte en un objeto de estudio respetable de la antropología, pues en ella se crean situaciones que pueden modelar una nueva sociedad:

[The communitas] can generate and store a plurality of alternative models for living, from utopias to programs, which are capable of influence the behavior of those in mainstream social and political roles in the direction of radical change, just as much as they can serve as instruments of political control (p. 33).

Para nuestro análisis es fundamental aprehender la distinción de Turner entre "liminar" y "liminoide". Lo "liminar" es una obligación dentro de las sociedades con solidaridad orgánica, es decir, un rito de paso que reincorpora a todos aquellos (a la gran mayoría) que participaron en el ritual. Lo "liminoide", por el contrario, es una opción, dentro de las sociedades industriales, tomada por quienes pretenden alejarse de las normas de la sociedad en forma permanente, decisión que encierra en sí el peligro de la muerte. En palabras del propio Turner: "One is play and choice, an entertainment, the other is a matter of deep 
seriousness, even dread" (Turner, 1982, p. 43). ${ }^{5}$ No estamos entonces frente al "rito de paso", cuya secuencia sigue los principios expuestos anteriormente y tiene una resolución, sino frente al "estado liminoide", donde permanece el individuo indefinidamente y que finalmente genera la "antiestructura".

La communitas presenta gran cantidad de analogías con eso que definimos como "suciedad". Turner (2008) muestra que uno de los ejemplos más impresionantes de esa analogía de la communitas se presenta en la forma de la indigencia, de los hombres santos que deciden usar vestimentas baratas (o no usar nada en absoluto), que dejan sus cabellos crecer sin nunca bañarse, acercándose así a lo meramente humano, despojándose de los harapos impuestos por la estructura de la sociedad. La liminaridad de la communitas también puede ser asimilada a un momento de la vida social en el cual se confrontan las actividades sin estructura, y sus consecuencias posibilitan a los hombres su más alto grado de autoconsciencia: es lo poco común, lo paradójico, lo ilógico y hasta al mismo tiempo lo perverso que estimula el pensamiento y genera preguntas (Turner, 2008, p. 238).

El estudio de las relaciones entre la estructura y la communitas puede ayudarnos a establecer los límites de cada una. La lingüista búlgara Julia Kristeva pretende ir más allá al intentar entender lo que estas personas representan:

No es por lo tanto la ausencia de limpieza o de salud lo que vuelve abyecto, sino
aquello que perturba una identidad, un sistema, un orden [...]. En la modernidad
occidental, y en razón de la crisis del cristianismo, la abyección encuentra resonan-
cias más arcaicas, culturalmente anteriores al pecado, para alcanzar su estatuto bí-
blico e incluso el de la impureza de las sociedades primitivas (Kristeva, 1989, p. 11).

Las reflexiones de Kristeva sobre lo que llama la abyección generan una idea de lo sagrado relacionada con los habitantes de la calle, idea subrepticia en Turner y ampliamente presentada por Mary Douglas (1973) en su obra Pureza y peligro. La noción de inmundicia que tiene cada sociedad está insertada en una red de símbolos y es una forma siempre relacionada al desorden y al caos que necesitan estar perfectamente confinados y restringidos.

Veamos dos ejemplos de autores que han trabajado con habitantes de la calle y vendedores de estupefacientes en Estados Unidos. Philippe Bourgois (1999; 2003), quien vivió en un barrio de vendedores de crack y heroína en Nueva York, afirma que la calle se constituye en un fórum alternativo, donde las personas pueden encontrar una "dignidad autónoma" frente a la exclusión de la sociedad y como reacción a la sumisión. La cultura de la calle es un universo de oposiciones políticas no coherentes, es una serie de prácticas rebeldes con un estilo antagónico y beligerante. Joanne Passaro (1996) estudió los lugares de venta de estupefacientes, así como las pandillas y los habitantes de la calle. La autora concluye que ellos desafían la naturalización política de las ideas tradicionales sobre el género y la clase, que asumen a la familia nuclear como el paradigma de la estabilidad y el buen funcionamiento de la sociedad. Como Bourgois, la antropóloga norteamericana afirma que existen asociaciones de la vida en la calle con el vagabundeo y la enfermedad mental; recordemos que en Estados Unidos

5 Uno es juego y elección, un entretenimiento, el otro es un problema de profunda seriedad, incluso de terror. 
las encuestas de salud mental están realizándose hace décadas, en las cuales esta asociación es "evidente". Para esta autora, los promotores de políticas públicas muchas veces olvidan que la vida en la calle es una opción tomada libre, activa y racionalmente: los hombres, en su mayoría, intentan huir así de los imperativos del padre proveedor y de la masculinidad exitosa estadounidense.

Sin embargo, en Colombia no siempre es la desilusión de la sociedad, sino la violencia dentro de la familia o el desplazamiento forzado lo que lleva a las personas a la calle (Barrios et al., 2006).

\section{La violencia y la vida en la calle: el caso del Cartucho}

Así como un reconocimiento de las representaciones sociales de los habitantes de la calle puede alimentar los discursos de los funcionarios y de los propios indigentes, las formas como estas personas llegan a la calle puede ser mejor aprehendido con su propia narración. De nuevo, aunque existen monografías que describen este "rito de paso", expondré en este texto extractos de las entrevistas realizadas para la investigación realizada para el ICBF (Barrios et al., 2006) sobre niños y niñas de la calle. En general, dentro de las entrevistas los menores mencionaron las principales causas de salida de la casa y de su entrada en la calle. Muchos de ellos relataron que su llegada a la calle se dio principalmente como consecuencia de la pobreza de sus familias; algunos de ellos pertenecen a la segunda generación en la calle. A esto se añade el abuso de drogas o de alcohol de sus parientes. La casa fue en muchos casos el "factor de expulsión" por violencia y abuso sexual, particularmente en hogares muy pobres con padrastros como jefes de familia.

De manera similar a otras historias centradas en el Cartucho (Cortés, 1994; Martínez, 2000; Rozo y Cadena, 2006), los jóvenes entrevistados, quienes habían sido abandonados o expulsados a la calle, tenían que aprender a defenderse rápidamente; la llegada a la calle como experiencia de libertad total significaba para el joven disponer de su propio cuerpo a voluntad, lo que se manifiesta también en la precocidad sexual y el abuso de sustancias psicoactivas. Sin duda, la calle es relatada como un territorio "salvaje" y agresivo, que requiere ser domesticado, adoptando sobre todo una actitud hostil y temeraria, usando un lenguaje cargado de argot y groserías: "Lo más difícil fue acoplarme allá [la calle], porque era un medio muy agresivo y yo me fui volviendo como ellos, por cualquier cosa me "regaba" [empezar a insultar sin contenerse] y venga démonos [golpes] y yo ya me volví asi" (Barrios et al., 2006, p. 79). A pesar de la violencia del nuevo ambiente, parece que son pocos los jóvenes que realmente desean volver a sus casas. En la calle, en medio del sufrimiento diario, encuentran un espacio vital de libertad. Se rompe así la dicotomía del sentido común casa-seguridad/ calle-peligro, lo que posiciona a la violencia intrafamiliar como un verdadero problema de salud pública, enmascarado por la espectacularización y publicidad que tienen los crímenes en la calle (Barrios et al., 2006; Jimeno, Góngora, Martínez y Suárez, 2005).

La llegada a la calle implica para los niños la entrada a un mundo nuevo, que en este trabajo se asemeja a la antiestructura. Esta antiestructura, como es definida por Turner (passim), no es necesariamente el reino del caos; discutiré, por 
lo tanto, la presentación que hace Turner de esta etapa de "drama social" como si fuera un momento de total homogeneidad y simetría entre sus miembros. Algunos autores brasileros, como Arno Vogel (1996), hacen este tipo de lectura de las comunidades que se forman en la calle, consideradas asimismo como una communitas, una "fraternidad que reúne sus miembros en los peligros y peripecias comunes de la salida para el mundo de la calle" (Vogel, 1996, p. 147), y cuya ley esencial es el silencio, seguida del principio que este autor llama de "no interferencia/no intromisión", es decir, dejar que cada cual haga su voluntad desde que no comprometa la integridad del grupo. Vogel arriesgó comprender a los "pivetes", como se designa comúnmente a los niños de la calle de Río de Janeiro, mediante el aparato teórico de Turner, de lo cual resultó una comunidad de "jóvenes libres" cuyo mayor temor era la policía militar. El artículo de Vogel se publicó en el marco de un evento traumático para los menores de la calle cariocas y que cuestionaba la violencia contra esta población, pero que dejó muchos elementos por fuera, como la propia acción de la Policía. En la "Chacina da Candelaria" ocurrida en 1993, la Policía militar asesinó a ocho muchachos que dormían en el exterior de la iglesia de la Candelaria, en el centro de la ciudad. La sevicia de la matanza propició una serie de investigaciones para comprender el fenómeno de los habitantes de la calle y la "limpieza social" en la ciudad, entre ellas la profunda etnografía de Silva y Milito (1994) con los sobrevivientes de esta masacre. Estos autores muestran que esta comunidad idílica y horizontal planteada por Vogel haciendo una lectura particular del término communitas no existe, y que la entrada a la "vida en la calle" es peligrosa y está además dominada por la propia Policía. Una antiestructura que, paradójicamente, está controlada por los agentes del Estado, especialmente el cuerpo de Policía.

Existen diferencias entre la estructura y el Estado que deben ser resaltadas. En este ensayo sigo la propuesta de Michel Foucault, para quien el Estado es una forma de gobernar, y la acción de Estado, el proceso de institucionalización, y cuya característica es la continua mudanza para hacer frente a la contingencia, mediante la sucesión de cambios discontinuos dentro de las formas del discurso, las maneras de nombrar y los mecanismos de acción de gobierno:

\footnotetext{
It is the tactics of government, which make possible the continual definition, and redefinition of what is within the competence of the state and what is not; thus the state can only be understood in its survival and its limits, on the basis of the general tactics of governmentality (Foucault, 2006, p. 143) ${ }^{6}$.
}

En este caso, los límites de la acción del Estado pueden aprehenderse a partir de las intervenciones frente a los habitantes de la calle en Bogotá.

Por otro lado, en una investigación que realizamos entre 2006 y 2007 (Góngora y Suárez, 2008) expusimos cómo era percibida la violencia dentro de un desaparecido lugar de Bogotá, la Calle del Cartucho. En esta zona estaban concentrados los mercados ilegales de estupefacientes y armas, las actividades ilícitas de prostitución y venta de objetos robados, junto con estructuras

6 Son las tácticas de gobierno, que hacen posible la definición continua y la redefinición de qué está dentro de las competencias del Estado y qué no lo está; así, el Estado solo puede ser entendido en su sobrevivencia y límites, sobre las bases de las tácticas generales de gobernamentalidad. 
criminales y la mayor cantidad de homicidios de la ciudad; además, estaba caracterizada por la mayor presencia de habitantes de la calle en la ciudad (Cámara de Comercio de Bogotá, 1997; Niño et al., 1998; Llorente, Escobedo, Echandía y Rubio, 2001; Robledo y Rodríguez, 2008).

Estas investigaciones concluían que, en general, la Calle del Cartucho era el summum del miedo, el peligro y la muerte en la capital. Es decir, en los términos de Turner, nos encontramos en presencia de un lugar en la ciudad reservado para esta población tan especial que estoy caracterizando. Más que eso, este lugar corresponde con aquel definido por Turner: la communitas, que en principio no tiene fronteras, en la práctica histórica ha sido limitada a regiones geográficas y a aspectos específicos de la vida social (Turner, 2008, p. 250). Si tenemos en cuenta que casi dos tercios de la población de habitantes de la calle de Bogotá pernoctaba y comercializaba estupefacientes en la Calle del Cartucho, junto con los discursos que se referían a los habitantes de la calle cuyo hábitat era la calle del Cartucho, es posible establecer una relación metonímica entre ellos, donde se toman los atributos del lugar como los atributos de la persona y viceversa.

Con base en esta reducción metonímica se justificaron las políticas de renovación urbana, que apelaron a la destrucción del hábitat y no a la transformación de los habitantes de la calle (Suárez, 2010). Existía una relación entre el miedo, el exceso del uso de la violencia y la Calle del Cartucho. Tal uso tenía, como mostré en las investigaciones mencionadas, dos aspectos fundamentales, uno interno y otro externo (Góngora y Suárez, 2008; Suárez, 2010). Me detendré primero en el uso interno, para entender mejor el funcionamiento social de este lugar que, como vimos, era el recipiente de la communitas o de la antiestructura en Bogotá. La violencia, y más exactamente la muerte (o su otra forma, el destierro), era la manera como se regulaban las relaciones sociales dentro de la Calle del Cartucho. Se ejercía un control excesivo, mediante la ley del silencio, en caso de que alguien presenciara un acto criminal, claramente opuesto al deber del testigo presente dentro de la denominada estructura. Sin embargo, las entidades de control, particularmente la Policía, no eran ajenas a este fenómeno: era por la violencia que, además de la estructuración de las jerarquías sociales, se legitimaban los agentes estatales.

¿Cuál era esa nueva jerarquía presente dentro de la antiestructura? Aquella determinada por la criminalidad y las acciones ilegales, es decir, en la cúspide visible aparecía el traficante, seguido del vendedor de estupefacientes junto con el cliente de clase media y alta, después los ladrones y las prostitutas del lugar y en la base, los habitantes de la calle, los recicladores, los "parias urbanos" (Waquant, 2006). Como nos encontramos frente a la antiestructura, estos personajes tienen una denominación especial. Turner muestra que dentro de la antiestructura las formas de referirse a las cosas cambian, creándose un lenguaje nuevo. En este caso, podemos citar así: el traficante es el "duro", el vendedor de drogas es el "jíbaro", el cliente adinerado es el "bacán", los ladrones son las "ratas" y finalmente los habitantes de la calle son los "desechables"; por otro lado, los agentes de control o policías son llamados "tombos". El control por el tráfico de drogas atraviesa toda la jerarquía y demuestra que, lejos de ser un momentum de alegre comunidad de cooperación mutua, como la describe Vogel (1996), en la antiestructura se establece un orden que huye de la lógica de "nosotros, los 
normales", según las palabras de Goffman (1975). Esta afirmación es confirmada por Llorente et al. (2001) en su artículo "Violencia homicida y estructuras criminales en Bogotá", que concluye que los homicidios cometidos en esta área de la ciudad, la Calle del Cartucho, se manifestaban principalmente en la forma de peleas espontáneas y muertes en manos de asesinos pagados, pero en el fondo se trataba de ajustes de cuentas, por ejemplo la pérdida de una "bomba", como se le llama a un paquete con 100 dosis de bazuco, o el no pago de una deuda, que en el lenguaje de la calle se llama "liebre", que es lo peor que puede suceder en este lugar; aquel que tenga una "liebre" será cazado y perseguido hasta que pague o muera. Claro está, los agentes de control del Estado no son ajenos a esta pirámide de poder; actúan como reguladores del comercio ilegal y de la prostitución, sirviendo de hilo conductor entre estas actividades ilegales como mediadores entre la estructura y la antiestructura. Este papel de la Policía será ampliado dentro del segundo modelo de transformación que explicaré más adelante.

La práctica de la violencia, y especialmente de la muerte, tiene elementos pedagógicos dentro de esta communitas o antiestructura. Brevemente, podemos ver un ejemplo de esta práctica que sigue, además de su intención reguladora, una forma de enseñanza particular que, como menciona Turner (1982), está relacionada con la monstruosidad, la exageración y lo traumático. ¿Cuán serio y atemorizante puede ser este aprendizaje de, por ejemplo, faltar a una deuda o hablar demasiado olvidando la ley del silencio? Hubert Ariza, en su narración sobre las evidencias de la destrucción de la Calle del Cartucho muestra las múltiples formas que adquiere esta muerte:

Los muertos eran echados a las alcantarillas, incinerados, enterrados en los patios de las casonas abandonadas, tirados al contenedor de la basura de la calle $9^{a}$, despedazados con motosierra por El Carnicero o dejados en los barrios vecinos para no boletear [hacer conspicua] más la zona (Ariza, 2007).

Puede apreciarse la sevicia y las diferentes alteraciones tanatológicas del cadáver: la muerte es llevada más allá, el cuerpo es inscrito con crueldad para dejar marcas indelebles en las personas de la zona, para aleccionar lo que le espera a quien rompa la ley del silencio o permanezca con una deuda. A esto se suma dejar carteles sobre los cadáveres despedazados: "por sapo, por soplón y por faltón" (Ariza, 2007). Otros actos más crueles son descritos por testigos sobrevivientes de la Calle del Cartucho:

\footnotetext{
En la época del 5 a 0 [partido de fútbol entre Colombia y Argentina celebrado en 1993] había una pandilla, eran unos animales espantosos, eran hermanos... Estas Pirañas negociaban con dulce o sal [bazuco o cocaína], estos tipos vendían sal y no admitían crítica, ellos tenían su rutina especial en la forma de sacrificar a la gente. Todos tenían un chuzo hecho con radios de moto, les sacaban punta, tenían una puntería tremenda. Los clavaban en el corazón (Góngora y Suárez, 2008, p. 131).
}

Otros utilizaban cuchillos untados con excrementos para asegurar la muerte de la víctima. Por medio de estas intervenciones se marcaban los espacios dentro de la Calle del Cartucho, se delimitaban los territorios, pero también se denotaba el perpetrador de la muerte para que, dentro de una lógica espectacular, 
todos supieran quién fue alcanzado por la ofensa o la traición, ganando así respeto y poder; en fin, dejando claro con cuál grupo el muerto rompió su trato.

\title{
4. La máquina correctiva en acción
}

\subsection{Primer modelo: higienización y estandarización}

El primer paso para incluir a los habitantes de la calle dentro de la sociedad, es decir, despojarlos de su condición "liminoide" y aparentemente "voluntaria" es mediante el procedimiento del "trabajo de la fachada”, como llamaría Goffman. En este caso, los individuos son elementos susceptibles de transformación. Nuestro ejemplo más claro de este cambio de la fachada del individuo es suministrado por el periódico El Tiempo, que describe perfectamente las acciones masivas del Estado sobre los cuerpos en términos de higienización, cambio de vestuario y donación de "comida decente":

\begin{abstract}
Para lograr que los habitantes de El Cartucho estuvieran limpios y peluqueados, la Policía organizó una jornada de salud. El Cuerpo de bomberos llevó una de las mangueras para echar agua como una regadera, desde el balcón interno de un tercer piso de una edificación, en la calle 9. Por ese baño pasaron niños, madres, ancianos y jovencitas de El Cartucho. Varios de ellos pudieron así lavarse el hollín y la mugre pegada de 30 y más días. Seguidamente les dieron una muda de ropa: camisa, pantalón, zapatos y saco. Varios de los habitantes pasó a recibir el desayuno gratuito ofrecido: tamal, chocolate o agua de panela. Los habitantes de El Cartucho durante los tres días, también tendrán almuerzo y comida gratis. Así transcurrió el primer día de fiesta, dedicada a la higiene y la salud (El Tiempo, 2000).
\end{abstract}

Los agentes de control del Estado, la Policía, fueron apoyados por el Cuerpo de bomberos, que podrían ser voluntarios u oficiales (adscritos a la Alcaldía Mayor), lo que no es especificado por el artículo. Queda claro que estas acciones del gobierno son recurrentes, puesto que la administración distrital repitió ese mismo procedimiento cuatro años después:

Los indigentes fueron divididos en hombres, mujeres y niños (150 en total) y empezó el primer paso: desnudarse para el baño. Las ropas que tenían puestas fueron incineradas y a cambio recibieron prendas nuevas. Después de la ducha, en la que colaboró el cuerpo de bomberos, llegó la peluqueada y en casos extremos, el exterminio de los piojos. Luego con cara nueva, pasaron a la revisión médica y odontológica. Se calzaron muelas, otras tocó sacarlas y los que tenían dolencias menores recibieron medicamentos gratis. Vino entonces un refrigerio, luego servicio de sastrería y zapatería, y el almuerzo (El Tiempo, 2004).

Dentro de la visión del Estado (y de la estructura que representa) un cambio en la fachada parece implicar instantáneamente una mudanza en los hábitos de estas personas o, por lo menos, algunos momentos de "normalidad". Es decir, no existe en el fondo de esta política de la "fachada" un cambio estructural en el individuo, sino simplemente de semblante. De modo que la limpieza le quita temporalmente esa connotación de peligro que involucra lo sucio. La operación de limpieza, en la cual los habitantes de la calle son desnudados en multitud y bañados con mangueras a presión, mientras que sus ropas son destruidas, recuerda 
el trato a personas excluidas totalmente, como sucede con los prisioneros de las cárceles y como sucedió con los judíos y minorías étnicas en los campos de concentración durante la guerra. La higienización de esta población, considerada "vulnerable", atenta contra su propia intimidad, a pesar de que la finalidad sea darles una "cara normal". Luego, el cuerpo de esta "nueva cara" es revisado por el personal médico. Tales intervenciones médicas han sido realizadas en los últimos años en la capital y otras ciudades de Colombia mediante brigadas itinerantes, lo que no estaba bien establecido en la década del 2000 como obligación de los hospitales públicos (Barrios et al., 2006). Después de esta intervención de "estandarización", los habitantes de la calle continúan con sus vidas, alejados de las preocupaciones cotidianas de la lógica económica.

Una de las conclusiones de las investigaciones para el Ministerio de la Protección Social (UNAL-Minprotección, 2007) apuntaba a que solo $10 \%$ de los habitantes de la calle permanecían hasta el final de los procesos de "resocialización", es decir, los procesos para inscribir una serie de prácticas de autocuidado y de autodisciplina sobre el cuerpo como unidad discreta. Frente a esta fracción de personas "resocializadas", de las cuales unas pocas consiguen empleos estables, la mayoría de los habitantes de la calle eran (y son) usuarios de los diversos programas de alimentación y de chequeo médico-odontológico gratuitos, que trabajan generalmente como brigadas móviles de médicos, odontólogos y trabajadores sociales. La mayoría de las instituciones que trabajan para su "restitución a la vida" (como si estuvieran muertos) procura convertirlos en "ciudadanos de bien", generalmente en instituciones que los aíslan o donde se prohíbe el consumo de estupefacientes y alcohol. Como resultado, las únicas relaciones posibles con el mundo que les ofrecen estas instituciones son el trabajo asalariado y el sustento de una familia, es decir, de acuerdo con la reflexión de Passaro (1996), entrar en el comportamiento del modelo androcéntrico del "male breadwinner". No obstante, los registros de egresados de los programas-terapias de las instituciones, tanto públicas como privadas y religiosas, son ínfimos comparados con la población de habitantes de la calle en la ciudad.

\subsection{2. Segundo modelo: eliminación}

Además de estas estrategias de reeducación del cuerpo, existe la eliminación propiamente dicha. La muerte, como enseñó Van Gennep (1978) un siglo atrás, es también un ritual de paso. Tales medidas de aniquilación de los cuerpos, que pueden ser leídas como otra forma de transformación, o por lo menos de sacar a las personas de la antiestructura, son llevadas a cabo en Colombia por la llamada "limpieza social", descrita vigorosamente por el investigador Carlos Rojas (1994) en su libro La violencia llamada limpieza social. Él concluye que estas medidas no pretenden solucionar esta problemática, sino prevenirla mediante la censura, por medio de la muerte, de una serie de comportamientos. El autor narra: "En mayo de 1989, en inmediaciones del Estadio El Campín, fue encontrado el cadáver de un niño de la calle con 3 impactos de bala en el estómago, uno en la cabeza y la boca sellada con el pegante que usaba para chupar" (Rojas, 1994, p. 62).

Estas descripciones sobre los cuerpos no están muy alejadas de aquellas que vimos para mantener el orden particular de la antiestructura, como señales de 
advertencia. No obstante, la "limpieza social" ataca tanto a los habitantes de la calle (a veces también prostitutas, travestis y ladrones, poblaciones que no voy a analizar en este texto) como los criminales en potencia, que pueden ser los jóvenes habitantes de asentamientos precarios de la periferia de la ciudad. Estos jóvenes bogotanos nos dieron una pista al reconocer el papel central que tiene la Policía en estos casos de violencia, lo que obviamente es negado insistentemente por los agentes del orden. La tesis de Lovisa Stannow (1996) sobre la "limpieza social" en Bogotá desarrolla una descripción de las diferentes mecánicas de este fenómeno:

In the first dynamic, which I described as "semi-spontaneous," the violence is carried out mainly by on-duty police, and sometimes by private security guards. These attacks are often perpetrated with the aim of controlling crime or punishing street people who have failed to fulfill their "duties" towards state agents. In the second dynamic, which I called "mercenary" violence, the perpetrators often belong to "death squads" which receive money and other backing to carry the "cleansing." While the actual hitmen are usually off-duty and ex-police officers and off-duty private security guards, members of civic groups called Juntas de Acción Comunal, and criminal elements (Stannow, 1996, p. 127)

El bajo estatus atribuido y el desprecio con que se trata a las víctimas de la "limpieza social", como se ha explicitado en este texto, hacen de ellas blancos fáciles. El periódico El Tiempo acompañó un caso de "limpieza social", solamente porque el crimen fue flagrantemente cometido por miembros activos de la Policía Metropolitana de Bogotá, de forma similar a la mencionada "Chacina da Candelaria" en Río de Janeiro, y sucedió dentro de las instalaciones de la Estación 24. Cito en extenso dos diferentes ediciones del periódico que hicieron referencia al caso:

El caso de Perea conmovió la opinión pública porque fue ultimado por el policía Orozco, después de ser capturado con otros tres niños, de 12, 14 y 16 años, el 3 de octubre del 2000, en el barrio Santa Fe [...] Varios cuerpos, incluido el de Perea, fueron encontrados en un contenedor de basuras, ubicado en la calle 8 con carrera 12 (El Tiempo, 2001).

A Over Steven Perea lo cogieron, le abrieron las manos y le pegaron entre todos. Después nos acostaron en el piso mojado para que nos revolcáramos en un charco, nos insultaron, patearon, amenazaron con las armas y, otra vez, hicieron la ruleta rusa [...] Según el relato de las víctimas, luego de llegar al parqueadero, jugar con ellos a la ruleta rusa, torturarlos y golpearlos, se hizo presente en el lugar un uniformado que, según las investigaciones, prestaba el servicio de guardia en el Instituto de Medicina Legal. El expediente de la Procuraduría revela que mientras los jóvenes

7 En la primera dinámica, que describí como "semi-espontánea", la violencia es llevada a cabo principalmente por policías en servicio, y a veces por guardias privados de seguridad. Estos ataques a menudo son perpetrados con el objeto de controlar el crimen o castigar a la gente de la calle que ha fallado en cumplir sus "deberes" hacia los agentes del Estado. En la segunda dinámica, que llamé violencia "mercenaria", los perpetradores a menudo pertenecen a "escuadrones de la muerte" que reciben dinero y otros apoyos para llevar a cabo las "limpiezas". Mientras que los verdaderos sicarios son usualmente expolicías o guardias de seguridad y oficiales fuera de servicio, los principales instigadores son civiles, que incluyen negociantes, miembros de grupos cívicos llamados Juntas de Acción Comunal y elementos criminales. 


\begin{abstract}
yacían en el piso, uno de los patrulleros gritó: Ahí viene el matón. Se trataba del agente Jhon Harold Orozco Díaz, quien con frialdad se acercó al niño Over Steven, le puso en la frente la ametralladora Uzi de dotación oficial y, sin mediar palabra alguna, le disparó. De inmediato, dice el expediente, los patrulleros salieron a correr y solo quedó Orozco Díaz con el niño muerto en el piso. El uniformado llamó a un indigente, identificado como Lucas Eduardo Fonseca Cabrera, y le entregó 20 mil pesos para que comprara un machete y unas bolsas plásticas (El Tiempo, 2003).
\end{abstract}

Este caso también es esclarecedor del modo como puede ser tratado el cuerpo despreciable: no basta con la simple muerte, ella tiene que ser ejecutada con sevicia, como para conjurar ese mal encarnado en estas personas. Aunque la mayoría de las muertes de habitantes de la calle dentro del Cartucho podrían atribuirse al arreglo interno de esta "antiestructura", la "limpieza social" debe ser entendida como un mecanismo diferente, como una relación explícita entre la "estructura" y la "antiestructura". La tesis de Stannow coloca en el centro de la distinción la participación de los agentes (o exagentes) de las fuerzas armadas del Estado y, a pesar de ser una práctica ilegal, es legítima para algunos uniformados. La práctica inicial sobre los jóvenes habitantes de la calle se asemeja al suplicio, como descrito magistralmente por Foucault (2004) en su obra "Vigilar y castigar". El suplicio no busca en este caso la confesión, como sería la tortura, sino brindar un espectáculo pedagógico. Si bien el castigo del suplicio monárquico tuvo translaciones, anotadas por el filósofo, que derivaron en la invención del panóptico, la práctica continuó marginalmente en la forma, por ejemplo, del linchamiento. La forma de matar y rematar, descrito igualmente por Stannow para identificar los casos de "limpieza social", pasa luego por un trabajo sobre el cadáver para hacerlo inidentificable, invisible: descuartizarlo y embalarlo.

Hasta cierto punto es posible diferenciar las prácticas tanatológicas, como descritas para mantener la jerarquía dentro de la antiestructura, y las realizadas por los agentes y exagentes del estado. Sin embargo, otras investigaciones han mostrado los vínculos entre las actividades ilícitas y estos agentes estatales, de modo que las fronteras entre la antiestructura y la estructura se diluyen precisamente en el eje articulador de ambas: el agente estatal. Por otra parte, y como sucede con el "modelo de estandarización" este no culmina con la eliminación efectiva de toda la población de habitantes de la calle. Se trata de asesinatos y desapariciones selectivos, que sigue patrones pedagógicos similares a los seguidos dentro de la antiestructura y que permiten que esta permanezca.

\title{
5. Consideraciones: el caos en la ciudad y el regreso al orden simbólico
}

Intentar un estudio del simbolismo de las relaciones sociales no implica pensar en términos de gradaciones evolutivas dentro de las sociedades contemporáneas. Por el contrario, es evidenciar la presencia de relictos religiosos dentro de esta era supuestamente posmoderna y posindustrial. Para entender las relaciones entre los habitantes de la calle y las instituciones debemos verlo dentro de los matices de las prácticas y menos en la política y el derecho, es decir, la lectura de la ley y del diagnóstico nos brindan una visión parcial. La propuesta inicial fue observar las representaciones sociales que tienen los funcionarios 
sobre los habitantes de la calle mediante el modelo propuesto por Goffman de "trabajo de la fachada". A la denominación "políticamente correcta" de esta población se añade una descripción que permite observar los contornos de los "habitantes de la calle" como representaciones. Aquí debemos diferenciar la construcción estadística de los habitantes de la calle que, si bien coincide en algunas características como el excesivo consumo de alcohol y de estupefacientes, muestra con mayor refinamiento las preferencias según grupos de edad o la distribución en la ciudad (Suárez, 2011). Luego de observar cómo se representan estas personas propuse seguir adelante con la propuesta simbólica para trabajar con los conceptos de Turner (passim), especialmente el de "liminoide" y "máquina correctiva". Señalé que la función ritual de la communitas en otras comunidades, donde se observan ritos de paso, difiere de la función simbólica del "liminoide" de las sociedades industriales. La ausencia de un "ritual de paso" público y que involucre a toda la comunidad en medio del regocijo en nuestra sociedad actual no implica la ausencia de "rituales de paso" que se encuentran escondidos y que se celebran cada vez en mayor intimidad. Varios habitantes de la calle (especialmente los jóvenes) describen en las etnografías estos "rituales de paso" para ingresar a la calle, la experiencia de violencia y de desorientación frente un nuevo ambiente agresivo.

La destrucción de El Cartucho para convertirlo en el Parque Tercer Milenio desplazó a los habitantes de la calle a la próxima calle del Bronx, que paradójicamente (o tal vez, como forma rigurosa de control de la antiestructura) quedaba al lado del Batallón de Reclutamiento del Ejército. De forma similar al Cartucho, la administración decidió "higienizar" el área de la calle del Bronx, despejando nuevamente y luego de una década a los "habitantes de la calle" quienes se integraron a "ollas" ya conocidas en la ciudad: Cinco Huecos (a espaldas del Colegio Agustín Nieto Caballero, de la red de instituciones públicas), las inmediaciones de la carrilera (calle 21) desde la Avenida Carrera 30 hacia el oriente, el lado sur del barrio Santafé (Calle 19 con carrera 16) y en el barrio Las Cruces.

Hasta el momento la sociedad colombiana presenta en forma general estas dos alternativas a los seres denominados "indeseables", los "parias sociales": la normalización a través de terapia-tratamiento, o la muerte en manos de la "limpieza social" ¿Qué otra alternativa puede ofrecerse a las personas desilusionadas con la sociedad y que pretenden otro tipo de formas de vida? Si seguimos igualando la mendicidad con la delincuencia, a pesar de las múltiples formas en que se imbrican ¿debemos entonces acabar con un "modo de vida" que simplemente no es acorde con las exigencias económicas de los tiempos modernos? Si vemos al habitante de la calle como un "espacio de disidencia" ¿la única respuesta que le ofrece la sociedad es abandonar su desilusión y reintegrarse a las formas alienadas de consumo? En el centro del debate sobre una nueva política de drogas se encuentra también la posibilidad de reconfigurar las relaciones que se han establecido con esta población, brindando otras alternativas de existencia que permitan, como bien dijo Turner, hacer explotar las energías creativas reprimidas dentro de la communitas.

\section{Referencias}


Ariza, H. (2007). "Yo tumbé el Cartucho con ayuda de Dios y una pistola". La ciudad jamás contada. Bogotá, Colombia: El Tiempo.

Barrios, M., Góngora, A. y Suárez, C. J. (Eds.). (2006). Derechos deshechos: Modelo de gestión para la garantía de los derechos sexuales y reproductivos de niños, niñas, adolescentes y jóvenes. Bogotá, Colombia: Universidad Nacional de Colombia - Instituto Colombiano de Bienestar Familiar.

Bourgois, P. (1999). Homeless en el barrio. En P. Bourdieu (Org.), La miseria del mundo (pp. 151-154). Madrid, España: Ediciones Akal.

Bourgois, P. (2003). In search of respect: selling crack. Cambridge, UK: Cambridge University Press.

Cámara de Comercio de Bogotá. (1997). Habitantes de la calle. Un estudio sobre la calle de El Cartucho en Santa Fe de Bogotá. Bogotá, Colombia: Cámara de Comercio de Bogotá.

Cortés, F. (1994). El espacio de la muerte en el Cartucho, Barrio Santa Inés, Santafé de Bogotá (tesis de pregrado). Universidad Nacional de Colombia, Bogotá, Colombia.

Douglas, M. (1973). Pureza y peligro. Un análisis de los conceptos de contaminación y tabú. Madrid, España: Siglo XXI Editores.

El Tiempo. (1 de marzo de 2003). Jugaron ruleta rusa con cada uno: Procuraduría. El Tiempo, pp. 1-6.

El Tiempo. (16 de noviembre de 2000). El Cartucho celebra su primer gran festival. El Tiempo, pp. 1-13.

El Tiempo. (17 de febrero de 2002). La amenaza de los cartuchitos. El Tiempo, pp. 1-9.

El Tiempo. (26 de febrero de 2001). Vinculan a indigente con muerte de niño. $E l$ Tiempo, pp. 1-9.

El Tiempo. (4 de noviembre de 2004). Agüita y jabón llegaron al Cartucho. El Tiempo, pp. 1-11.

Foucault, M. (2004). Vigiar e punir. Petrópolis, Brasil: Editora Vozes.

Foucault, M. (2006). "Governmentality". In A. Sharma \& A. Gupta (Eds.), The Anthropology of the State (pp. 131-143). Oxford, UK: Blackwell Publishing.

Goffman, E. (1975). A representação do eu na vida cotidiana. Petrópolis, Brasil: Vozes.

Goffman, E. (1980). Estigma: notas sobre a manipulação da identidade deteriorada. Rio de Janeiro, Brasil: Zahar Editores.

Góngora, A. y Suárez, C. J. (2008). Por una Bogotá sin mugre. Violencia, vida y muerte en la cloaca urbana. Universitas Humanística, (66), 107-138.

Jimeno, M., Góngora, A., Martínez, M. y Suárez, C. J. (2005). Manes, mansitos y manazos: una metodología de trabajo sobre violencia intrafamiliar y sexual. Bogotá, Colombia: Universidad Nacional de Colombia - Departamento Administrativo de Bienestar Social.

Kristeva, J. (1989). Poderes de la perversión. Ensayo sobre Louis-Fernand Céline. México D.F., México: Siglo XXI editores.

Llorente, M. V., Escobedo, R., Echandía, C. y Rubio, M. (2001). Violencia homicida y estructuras criminales en Bogotá. Análisis Político, (44), 343-375.

Martínez, P. P. (2000). Entre el pánico y el éxtasis: territorio en el Cartucho (tesis de pregrado). Universidad Nacional de Colombia, Bogotá, Colombia. 
Niño, S., Lugo, N., Rozo, C. y Vega, L. (1998). Territorios del miedo en Santafé de Bogotá. Imaginarios de los ciudadanos. Bogotá, Colombia: Tercer Mundo Editores.

Passaro, J. (1996). The Unequal Homeless: Men on the Streets, Women in their Place. New York, US: Routledge.

Robledo, A. M. y Rodríguez, P. (2008). Emergencia del sujeto excluido: aproximación genealógica a la no-ciudad en Bogotá. Bogotá, Colombia: Editorial Pontificia Universidad Javeriana.

Rojas, C. (1994). La violencia llamada "limpieza social". Bogotá, Colombia: CINEP. Rozo, A. y Cadena, C. (2006). Yo salí del infierno: sobreviviente del Cartucho. Bogotá, Colombia: CIEDPAZ.

Silva, H. \& Milito, C. (1994). Vozes do Meio Fio. Rio de Janeiro, Brasil: Editora Relume \& Dumará.

Stannow, L. (1996). "Social cleansing" in Colombia (tesis de maestría). Simon Fraser University, Burnaby, Canadá.

Suárez, C. J. (2010). Renovación urbana ¿una respuesta al pánico moral? Territorios, (22), 111-124.

Suárez, C. J. (2011). O Estado e o abjeto: Discursos, dispositivos e políticas públicas para a remoção de moradores de rua em Bogotá (tesis de maestría). Universidade Federal Fluminense, Niterói, Brasil.

Turner, V. (1982). From Ritual to Theater, the Human Serioussness of Play. New York, US: Performing Arts Journal Publications.

Turner, V. (2008). Dramas campos e metáforas: ação simbólica na sociedade humana. Niterói, Brasil: Editorial Universidade Federal Fluminense.

Universidad Nacional de Colombia (UNAL) - Ministerio de la Protección Social (MinProtección). (2007). Identificación, documentación y socialización de experiencias de trabajo con habitantes de y en calle. Recuperado de http://www. siamisderechos.org/banco/todo/ATT1359514894.pdf

Van Gennep, A. (1978). Os ritos de passagem. Petrópolis, Brasil: Vozes.

Vogel, A. (1996). Da casa à rua: a cidade como fascínio e descaminho. En A. Fausto y R. Cervini (Org.), O trabalho e a rua: crianças e adolescentes no Brasil urbano dos anos 80 (pp. 133-150). São Paulo, Brasil: UNICEF/FLACSO/UNESCO.

Waquant, L. (2006). Castigar los parias urbanos. Antípoda, (2), 59-66. 
